\title{
Photospheric Magnetic Field: Relationship Between North-South Asymmetry and Flux Imbalance
}

\author{
E.S. Vernova ${ }^{1}$ - M.I. Tyasto ${ }^{1}$ - D.G. Baranov ${ }^{2}$ \\ (C) Springer $\bullet \bullet \bullet \bullet$
}

\begin{abstract}
Photospheric magnetic fields were studied using the Kitt Peak synoptic maps for $1976-2003$. Only strong magnetic fields $(B>100 \mathrm{G})$ of the equatorial region were taken into account. The north-south asymmetry of the magnetic fluxes was considered as well as the imbalance between positive and negative fluxes. The north-south asymmetry displays a regular alternation of the dominant hemisphere during the solar cycle: the northern hemisphere dominated in the ascending phase, the southern one in the descending phase during Solar Cycles $21-23$. The sign of the imbalance did not change during the 11 years from one polar-field reversal to the next and always coincided with the sign of the Sun's polar magnetic field in the northern hemisphere. The dominant sign of leading sunspots in one of the hemispheres determines the sign of the magneticflux imbalance. The sign of the north-south asymmetry of the magnetic fluxes and the sign of the imbalance of the positive and the negative fluxes are related to the quarter of the 22-year magnetic cycle where the magnetic configuration of the Sun remains constant (from the minimum where the sunspot sign changes according to Hale's law to the magnetic-field reversal and from the reversal to the minimum). The sign of the north-south asymmetry for the time interval considered was determined by the phase of the 11-year cycle (before or after the reversal); the sign of the imbalance of the positive and the negative fluxes depends on both the phase of the 11-year cycle and on the parity of the solar cycle. The results obtained demonstrate the connection of the magnetic fields in active regions with the Sun's polar magnetic field in the northern hemisphere.
\end{abstract}

Keywords: Solar cycle; Magnetic field, Photosphere; North-south asymmetry

\section{Introduction}

The principal cause of all manifestations of solar activity is the magnetic field of the Sun, which, through the process of its development, displays a surprising symmetry. The magnetic field of the Sun displays the 22-year periodicity in two

\footnotetext{
1 IZMIRAN, SPb. Filial, St. Petersburg, Russian Federation email: helena@ev13934.spb.edu

2 A.F. Ioffe Physical-Technical Institute, St. Petersburg,

Russian Federation email: d.baranov@mail.ioffe.ru
} 
ways (see, e.g., Charbonneau, 2010). The first of the 22-year cycles is related to the global magnetic field of the Sun (as a magnetic dipole) with a change of sign every 11 years, after the solar-activity maximum. Another 22-year periodicity is connected with local magnetic fields (magnetic fields of sunspots). According to the Hale polarity law, at each minimum of solar activity, sunspots of a new cycle appear in each of the Sun's hemispheres with the sign of the leading sunspots opposite to the sign of the following sunspots in the same hemisphere and opposite to the sign of the leading sunspots of the other hemisphere. The signs of the leading and following sunspots remain constant up to the following minimum when sunspots of a new 11-year cycle appear with polarities opposite to the previous ones. Thus, the magnetic field of the Sun varies with a 22 -year periodicity that manifests itself both in the global magnetic field change, and in the change of local magnetic fields of the Sun.

There exist certain regularities in the changes of the global and the local magnetic fields during a 22-year solar cycle. When one considers the two magnetic cycles as a unique process, we see that in the course of a 22-year magnetic cycle there are moments when the relation between the polarities of the global and local magnetic fields changes. These moments are related either to the change of the Sun's global magnetic-field polarity during high solar activity, or to the alternation of the polarities of leading and following sunspots at the solar-activity minimum. Thus, during a 22-year cycle there are four intervals where the polarities of the global magnetic field and of the leading sunspots for each of the hemispheres are constant. These four intervals are from the solaractivity minimum to the magnetic-field reversal of the Sun and from the reversal to the next minimum in an even 11-year solar cycle, and analogously for the next odd cycle. The relations between the polarities of the global and local magnetic fields will repeat themselves in the next 22-year cycle. There is one remarkable feature that we used in addition to this: for all ascending phases of solar activity from a minimum to the reversal the sign of the global field always coincides with the sign of the leading sunspot in the same hemisphere: for the northern hemisphere for an even cycle these signs will be minus, and for an odd cycle the signs are plus.

Thus, seen on a longer time scale, the distributions of the local and the global magnetic field exhibit a symmetry with respect to the solar Equator. At the same time, an asymmetry is observed in the magnetic fields, that manifests itself in three different forms: as the longitudinal asymmetry, as the north-south asymmetry, and as the asymmetry of the leading and following sunspots in an active region.

We found it to be essential to divide a 22-year magnetic cycle into four intervals where the polarities of the local and global magnetic fields are constant when we considered the longitudinal distribution of sunspots (Vernova et al., 2004) and of photospheric magnetic fields (Vernova, Tyasto, and Baranov, 2007). The longitudinal distribution for the ascending phase and the maximum sharply differ from the longitudinal distribution for the descending phase and the minimum of the solar cycle. Active longitudes change by $180^{\circ}$ when we pass from the ascending to the descending phase. The maximum of the longitudinal distribution is reached at $180^{\circ}$ for the ascending phase and the maximum of the 
solar cycle when the polarities of the leading spot and of the global field coincide in each hemisphere, and at $0^{\circ} / 360^{\circ}$ for the descending phase and the minimum when these polarities are opposite.

An analogous pattern of the active longitudes was discovered for coronal mass ejections by Skirgiello (2005). In a manner similar to the results of Vernova et al. (2004) and Vernova, Tyasto, and Baranov (2007), two nearly antipodal longitudes dominated alternately: the domination of the longitude $180^{\circ}$ coincided with the ascending phase and the maximum, while the domination of the longitude $30^{\circ}$ coincided with the descending phase and the minimum of the solar cycle.

There also exists an asymmetry between the leading and following sunspots in an active region (Bray and Loughhead, 1964, Vitinsky, Kopecky, and Kuklin, 1986). Solanki (2003) in his survey considered the question: "Why are leading polarity spots in an active region often larger than following polarity spots?" as one of the important open questions. Asymmetry of the leading and following polarities of an active region are of interest and are discussed in many publications (see van Driel-Gesztelyi and Petrovay, 1990, Fan, Fisher, and DeLuca, 1993, Fan, 2009, and references therein).

Another aspect of the solar-activity asymmetry is the north-south asymmetry that was discovered when investigating various forms of solar activity, such as sunspots, flares, or sudden disappearances of solar prominences (see Carbonell et al., 2007, Verma, 2009, Sykora and Rybak, 2010, and references therein). It was observed that there are some common features in the behavior of the north-south asymmetry for different solar-activity indices (Vizoso and Ballester, 1990; $\mathrm{Li}$ and $\mathrm{Gu}, 2000)$. For example, during the period of the northern sunspot dominance, $78 \%$ of solar flares were observed in this hemisphere (Swinson, Shea, and Humble, 1986). However, it was shown by Roy (1977) that the northsouth asymmetry of solar flares does not follow the 11- or 22-year cycle of the occurrence of major flares. The north-south asymmetry of sunspot areas (see Ballester, Oliver, and Carbonell, 2005, and references therein) shows three significant peaks in the power spectrum with periods of $43.25,8.65$, and 1.44 years. For the north-south asymmetry of sunspot groups the long-term period has been found to be about 80 years (Waldmeier, 1957; : Pulkkinen et al., 1999) and about 110 years (Verma, 2009).

The north-south asymmetry is important for the topology of the interplanetary space, and it influences both interplanetary and near-Earth space parameters. For example, it affects the position of the heliospheric current sheet (see Mursula and Hiltula, 2003, Wang and Robbrecht, 2011, and references therein) and leads to a difference in the number of away-from-the-Sun and toward-the-Sun interplanetary-magnetic-field-sector days (Swinson et al., 1991). Since some mechanisms of cosmic-ray modulation depend on the current sheet, the north-south asymmetry of solar activity must be taken into consideration when investigating cosmic-ray modulation.

Of special interest in the framework of the present article is the change of the north-south asymmetry in the course of 11-year solar cycle. Newton and Milsom (1955) and Waldmeier (1971) noted that the North dominates during the ascending phase of the cycle while the South dominates during the declining phase. Vizoso and Ballester (1987) showed that for Cycles $18-21$ the sign of 
the north-south asymmetry of the sudden disappearance of solar prominences changed from positive to negative during the solar maximum at the time of the Sun's magnetic-field reversal. Yadav, Badruddin, and Kumar (1980) studied the north-south asymmetry of solar flares and showed that the majority of flares was concentrated in the northern hemisphere before 1970 and in the southern hemisphere after 1970 (the year of the reversal).

The heliographic distribution of significant $\mathrm{H} \alpha$ and $\mathrm{X}$-ray flares has been studied during the period 1975 - 1989 (Curto and Gaya-Pique, 2009), who found that the northern hemisphere dominates in the ascending part of each solar cycle and the southern hemisphere dominates in the descending part. Li and $\mathrm{Gu}(2000)$ collected 20 different solar-activity phenomena to investigate solar activity in Solar Cycle 21, and found that solar activity has a northern bias at first, and as the cycle progresses, southern predominance takes over. The pronounced north-south asymmetry in the distribution and the number of CMEs in Cycle 23 observed by the Solar and Heliospheric Observatory spacecraft was reported by Minarovjech, Rušin, and Saniga (2008): this asymmetry is in favor of the northern hemisphere at the beginning of the cycle, and of the southern hemisphere from 2001 (the year of the reversal) onward.

The behavior of the north-south asymmetry during the Sun's global magneticfield reversal is of special importance. The transition of the dominant role from the northern hemisphere to the southern one during the time of the reversal was found for sunspot area for Cycles 16 - 22 (Vernova et al., 2002) and for the solarflare index and solar-group area for Solar Cycles 21 - 22 (Kane, 2005). Vernova et al. (2002) studied the north-south asymmetry using a vector summation of the sunspot areas that reduced the stochastic, longitudinally evenly distributed sunspot activity and, therefore, emphasized the more systematic, longitudinally asymmetric sunspot activity. We found a systematic alternation of the dominant hemisphere during high solar-activity periods, which is reproduced from cycle to cycle. This effect was analyzed for Cycles $16-22$ by a superposed-epoch method using the date of magnetic reversal in the southern hemisphere as the zero-epoch time to obtain an average pattern of the north-south asymmetry. Kane (2005) discovered analogous patterns (although with slightly different lengths of the domination periods) for solar-flare index and solar-group area for Solar Cycles 21 and 22 .

The connection of north-south asymmetry with the phase difference of solar activity in the two hemispheres was established in many articles. Waldmeier (1971) has shown that because of a phase shift, the hemisphere preceding in time is more active on the ascending branch of the solar cycle, whereas on the descending branch the hemisphere following in time dominates. The phase shift changes its sign every four cycles so that the full period contains eight 11-year cycles. This periodicity was observed for Solar Cycles $10-20$.

Various methods were proposed to distinguish the two effects: real asymmetry, i.e. difference between the strength of the solar cycle in the two hemispheres, and phase asymmetry. By using the cross-recurrence plot technique it was shown that the north-south sunspot asymmetry is due to phase asynchrony between northern and southern hemispheric activities (Zolotova and Ponyavin, 2006. 2009). The persistence of phase-leading in one of the hemispheres exhibited a 
secular variation. Beginning from Solar Cycle 13 and up to the end of Cycle 16, the North was leading in time. After this epoch and until the beginning of Cycle 20, the situation was reversed. After Cycle 20 it was restored to the situation that was observed before Cycle 16. Later these results were extended for the period of 300 years as far back as to the Maunder Minimum (Zolotova et al., 2010).

Deng et al. (2013) have analyzed the phase asynchrony of hemispheric flare activity for the period of $1966-2008$. During this period, flare activity in the northern hemisphere developed six months earlier than that in the southern hemisphere. The empirical-mode decomposition showed that the main periodicities of flare activity in the northern hemisphere slightly differ from those in the southern one, which should also lead to phase asynchrony between them.

Another approach was used by Muraközy and Ludmány (2012), who represented the cycle profile by its centre of mass. For Solar Cycles $12-23$ the phase of the hemispheric cycles shows an alternating variation: the northern cycle led in four cycles and followed in four cycles. This period approximately corresponded to the length of the Gleissberg cycle. As pointed out by the authors, this periodicity may not be absolutely rigid if the mechanism controlling this variation is not part of the solar dynamo. Although this variation persisted for the last 14 solar cycles, it seems that phase relations are violated for Solar Cycle 24 (Svalgaard and Kamide, 2013) where the northern hemisphere again precedes the southern one, contrary to the expected change of domination.

The magnetic fluxes of the Sun and their imbalance have been studied by many authors on the basis of different data that characterize the magnetic activity. The problem of the nature of the imbalance is widely discussed in the literature. As stated by Kotov (2009), the theory cannot explain why the positive or the negative field dominates for one year or more practically over the whole Sun, or, more precisely, over the visible hemisphere. The observed prevalence of the negative mean magnetic field of the Sun as a star in 1968 - 1969 and $1989-1990$ could not be compensated by the northern field of the polar regions.

Choudhary, Venkatakrishnan, and Gosain (2002) studied the magnetic-flux imbalance of active regions using the longitudinal magnetograms obtained from the National Solar Observatory at Kitt Peak. The median value of the flux imbalance in 137 active regions was found to be about $9.5 \%$. The global-flux imbalance in the active latitude zone of $10^{\circ}-40^{\circ}$ is solar-cycle dependent. The flux imbalance is lower than $5 \%$ when the active zones located in both hemispheres are considered.

As demonstrated by these results, there are numerous manifestations of asymmetric features of solar activity and the Sun's magnetic fields. Further research is needed to combine all varieties of these phenomena within the framework of the generalized model of the solar dynamo.

The present article is concerned with the asymmetry of strong photospheric magnetic fields $(B>100 \mathrm{G})$ of the equatorial region (helio-latitudes from $-40^{\circ}$ to $+40^{\circ}$ ). We consider the north-south asymmetry of the magnetic fluxes as well as the imbalance between the positive and negative fluxes.

In Section 2 we describe the data and discuss the method applied in the article. Section 3 is devoted to the north-south asymmetry of the photospheric 
magnetic field. In Section 4 positive and negative magnetic fields and their imbalance in each of the solar hemispheres are considered. The imbalance of positive and negative magnetic fluxes for the whole latitude range from $-40^{\circ}$ to $+40^{\circ}$ is considered in Section 5, while in Section 6 we are concerned with the relations between the imbalance of the fluxes and the properties of the leading and following sunspots. In Section 7 we discuss and interpret the results obtained and draw our conclusions.

\section{Data and Method}

For this study we used synoptic maps of the photospheric magnetic field produced at the National Solar Observatory/Kitt Peak (available at nsokp.nso.edu). These data cover the period from 1975 to 2003 (Carrington Rotations 16252006). Because the data have many gaps during the initial period of observations, we included in our analysis the data starting from Carrington Rotation 1646. Synoptic maps have the following spatial resolution: $1^{\circ}$ in longitude (360 steps); 180 equal steps in the sine of the latitude from -1 (south pole) to +1 (north pole). Thus every map consists of $360 \times 180$ pixels of magnetic-flux values.

Strong magnetic fields of both polarities occupy a relatively small part of the Sun's surface. The magnetic-field strength for the period $1976-2003$ shows a nearly symmetric distribution with $98.7 \%$ of values in the $0-100 \mathrm{G}$ interval, whereas pixels with magnetic strength above $100 \mathrm{G}$ occupy only $1.3 \%$ of the solar surface. Even so, the total number of the latter pixels is large enough, amounting to $3 \times 10^{5}$ for $1976-2003$, and thus allowing detailed analysis of strong magnetic fields and their temporal changes.

We focused here on the photospheric magnetic fields connected with solar active regions. To do this, we limited our research to the near-equatorial and strong magnetic fields. From here on only equatorial magnetic fields are considered, i.e. helio-latitudes from $-40^{\circ}$ to $+40^{\circ}$. To calculate the magnetic flux only pixels with magnetic field strengths higher than $100 \mathrm{G}$ were selected. This approach allowed us to investigate changes of the photospheric magnetic field connected with active areas on the Sun, among which bipolar sunspot groups play an important role. For each synoptic map, magnetic fluxes for the northern and southern hemispheres were analyzed separately. Fluxes for positive and negative magnetic fields were calculated for each of the hemispheres. Thus, for each synoptic map four different characteristics of magnetic flux were obtained: $F_{\mathrm{N}}^{\text {pos }}, F_{\mathrm{N}}^{\text {neg }}, F_{\mathrm{S}}^{\text {pos }}, F_{\mathrm{S}}^{\text {neg }}$. For each solar cycle, positive and negative fluxes in the northern hemisphere $\left[F_{\mathrm{N}}^{\mathrm{pos}}\right.$ and $\left.F_{\mathrm{N}}^{\text {neg }}\right]$ coincide in sign with the fields of the leading or following sunspots. The same is true for the southern hemisphere where the polarities of the leading (following) sunspots are opposite to those of the northern hemisphere. It is expected that under our restrictions (strong magnetic fields of the sunspot zone) each of the four fluxes is connected with the sunspots of either leading or following polarity, but they are not identical to the sunspot fluxes. We studied the changes of these fluxes, as well as temporal changes of the difference between positive and negative fluxes. 


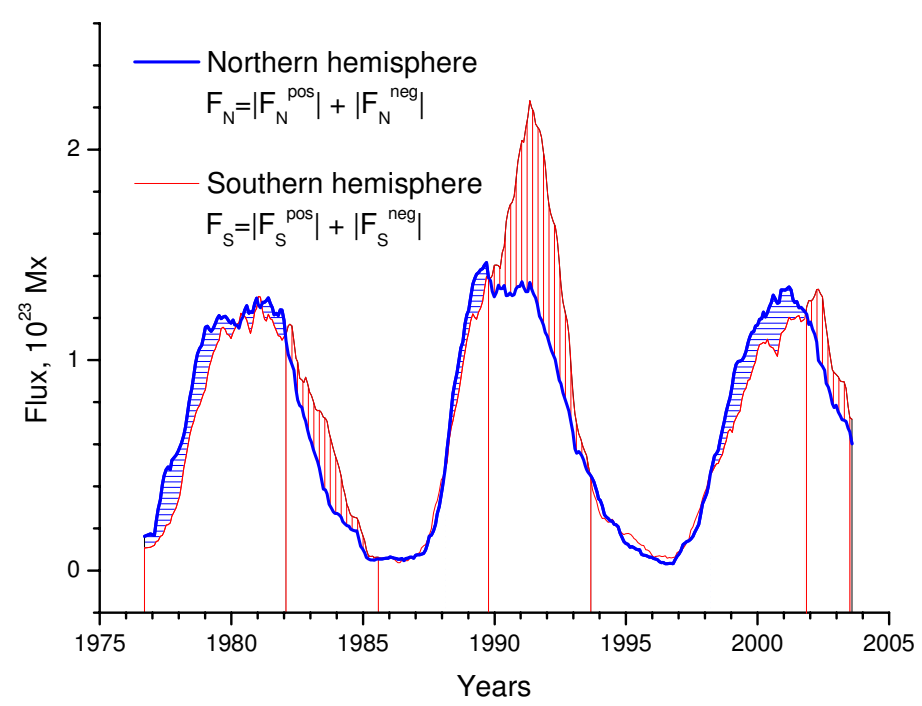

Figure 1. Photospheric magnetic flux: northern hemisphere $\left[F_{\mathrm{N}}=\left|F_{\mathrm{N}}^{\text {pos }}\right|+\left|F_{\mathrm{N}}^{\text {neg }}\right|\right]-$ thick blue curve; southern hemisphere $\left[F_{\mathrm{S}}=\left|F_{\mathrm{S}}^{\text {pos }}\right|+\left|F_{\mathrm{S}}^{\text {neg }}\right|\right]$ - red curve. Only strong magnetic fields $[B>100 \mathrm{G}]$ of the equatorial region (helio-latitudes from $-40^{\circ}$ to $+40^{\circ}$ ) were taken into account. Periods of northern hemisphere domination are marked by horizontal blue shading. Vertical red shading marks southern hemisphere domination. Vertical lines show the change of the dominant hemisphere.

\section{North-South Asymmetry of the Photospheric Magnetic Field}

We considered the total flux of the northern hemisphere (latitudes from $0^{\circ}$ to $\left.+40^{\circ}\right)$ as the sum of absolute values both of positive and negative fluxes $\left[F_{\mathrm{N}}=\right.$ $\left.\left|F_{\mathrm{N}}^{\mathrm{pos}}\right|+\left|F_{\mathrm{N}}^{\text {neg }}\right|\right]$ and the total flux of the southern hemisphere (latitudes from $0^{\circ}$ to $\left.-40^{\circ}\right)$ as $F_{\mathrm{S}}=\left|F_{\mathrm{S}}^{\mathrm{pos}}\right|+\left|F_{\mathrm{S}}^{\text {neg }}\right|$, respectively.

In Figure 1 total magnetic fluxes for the northern and southern hemispheres are shown for 1976-2003. To exclude random fluctuations, flux values were smoothed using adjacent averaging over 20 solar rotations. Both fluxes change following the 11-year cycle of solar activity. However, some differences between fluxes of the northern and southern hemispheres can be seen. During the ascending phase of the solar cycle, the northern hemisphere dominates, while during the descending phase the southern hemisphere always dominates the northern one. These features occur regularly throughout Solar Cycles $21-23$.

This result becomes more evident if one considers the difference of fluxes

$$
\Delta F_{\mathrm{NS}}=\left(\left|F_{\mathrm{N}}^{\mathrm{pos}}\right|+\left|F_{\mathrm{N}}^{\mathrm{neg}}\right|\right)-\left(\left|F_{\mathrm{S}}^{\mathrm{pos}}\right|+\left|F_{\mathrm{S}}^{\text {neg }}\right|\right),
$$

that is the north-south asymmetry of the magnetic field (Figure 2). The difference $\left[\Delta F_{\mathrm{NS}}\right]$ is close to zero during periods of low solar activity. In each of the solar cycles, the difference passes twice through zero: during the global magneticfield reversal and during the solar minimum. As a result, we see a regular change of alternately dominating hemispheres. In the ascending phase (from the solaractivity minimum to the reversal) the northern hemisphere always dominates 


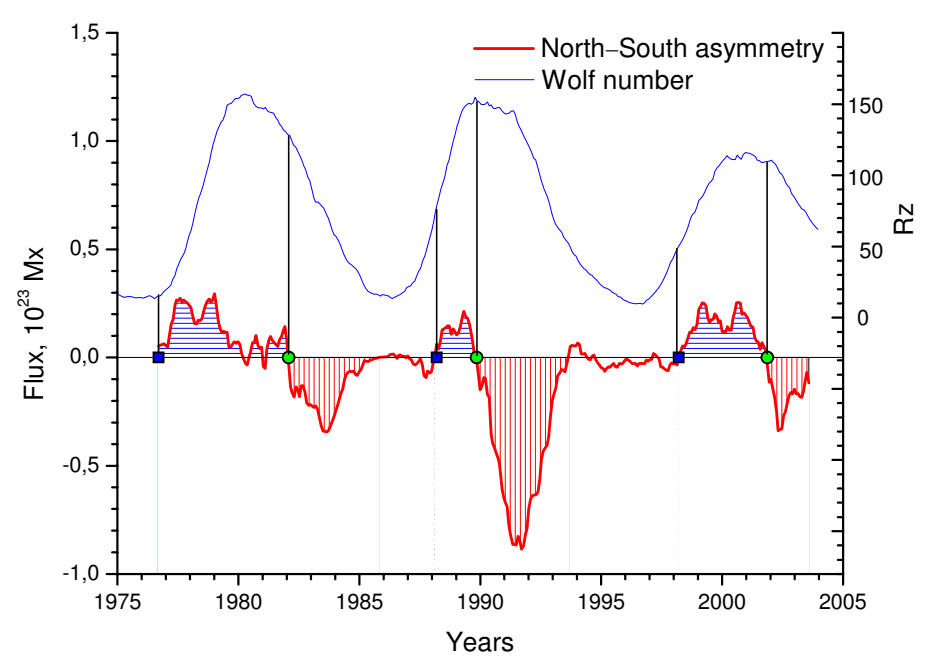

Figure 2. north-south asymmetry of the photospheric magnetic flux (thick red line): $\Delta F_{\mathrm{NS}}=\left(\left|F_{\mathrm{N}}^{\mathrm{pos}}\right|+\left|F_{\mathrm{N}}^{\text {neg }}\right|\right)-\left(\left|F_{\mathrm{S}}^{\mathrm{pos}}\right|+\left|F_{\mathrm{S}}^{\text {neg }}\right|\right)$. Periods of northern hemisphere domination are shown by horizontal blue shading; vertical red shading corresponds to southern hemisphere domination. Vertical lines show the change of the dominant hemisphere. Wolf numbers [Rz] (blue line) are plotted for comparison of magnetic flux changes with the solar-activity cycle. The change of the north-south asymmetry sign is marked by squares (ascending phase of the solar cycle) and by circles (period of the global magnetic field reversal).

(the difference has positive sign). During reversal, the difference becomes negative, i.e. the leading role passes to the southern hemisphere. Domination of the southern hemisphere continues from the reversal to the next minimum. Near the solar-activity minimum the sign of the difference changes again and the northern hemisphere becomes dominant.

Thus, we draw the following conclusions: we have considered the north-south asymmetry of the photospheric magnetic field for almost three solar cycles (Solar Cycles 21-23) and for each of them we have observed the same rule of the northsouth asymmetry change in the 11-year solar cycle. This rule can be formulated as follows: each of the 11-year cycles can be divided into two parts, the ascending phase and the descending phase of solar activity. For each of these parts, the following effects take place:

i) In the ascending phase the northern hemisphere always dominates the southern one;

ii) During reversal the dominant hemisphere changes so that in the descending phase to the solar minimum the southern hemisphere always dominates.

This effect cannot be seen in the north-south asymmetry averaged for longterm periods such as the 11-year cycle or longer. On the other hand, strong fluctuations mask the effect if one considers short time intervals. Yet these features are clearly seen after averaging of the data over 20 solar rotations. Maxima of the north-south asymmetry amount to $\approx 30 \%$ of the average magnetic flux for $1976-2003$. The close connection of the asymmetry with the solar-cycle phase is clearly seen during the three cycles. 
We emphasized in the Introduction that characteristic points of the 22-year magnetic cycle play important roles for the solar-activity development. These points are: i) reversal of the global magnetic field of the Sun (after the solaractivity maximum) and ii) change of sign of the leading and following sunspots in each of the hemispheres according to Hale's law (at solar-activity minimum). Between these points the sign of the global magnetic field and the sign of leading sunspots for each of the hemispheres is constant. Thus, for each quarter of the 22-year magnetic cycle the polarities of the global and local magnetic fields of the Sun remain constant.

As was shown, in the ascending phase up to the reversal, the magnetic flux of the northern hemisphere always dominates when compared with the flux of the southern hemisphere. At this time the leading sunspots of the northern hemisphere have the same polarity as the global field in the northern hemisphere. After the reversal, the sign of the global field in the northern hemisphere coincides with the sign of leading sunspots in the southern hemisphere. At the same time, we see that the dominant hemisphere has changed as well: domination passes to the southern hemisphere.

An important conclusion follows: leading sunspots of the dominant hemisphere have the same polarity as the global magnetic field in the northern hemisphere. It should be noted that these results are true for the period of Solar Cycles $21-23$ when the activity of the northern hemisphere preceded that of the southern hemisphere (see Introduction).

These results are summarized in Table 1. We present the 22-year period (Cycles 21 and 22), divided into four quarters. Commonly, two consecutive solar cycles are combined into a 22-year magnetic cycle, starting with an even one (Gnevyshev and Ohl, 1948). Because we have no data for the whole of Cycle 23 , we considered the odd-even pair of cycles. The first quarter of the 22year period includes the ascending phase of Cycle 21 from the minimum to the reversal; the second quarter is a period of the descending phase of Cycle 21 from the reversal to the minimum. Similarly, Cycle 22 is divided into two parts. For each of these quarters the following characteristics are shown: i) the global magnetic-field sign (northern hemisphere); ii) the dominant hemisphere; iii) the sign of leading sunspots of the dominant hemisphere. The regular change of the dominant hemispheres can be seen in Table 1 as well as the coincidence of the global magnetic-field sign in the northern hemisphere with the sign of the leading sunspots of a dominant hemisphere for each of the quarters.

These features of the north-south asymmetry of the magnetic field can be expressed by the following equation that establishes the law of the north-south asymmetry sign change for the two parts of the 11-year cycle - before the global magnetic-field reversal and after it:

$$
\operatorname{sign} \Delta F_{\mathrm{NS}}=(-1)^{\mathrm{k}+1}, \quad \text { where } \mathrm{k}=1,2
$$

( $\mathrm{k}=1$ corresponds to the interval from the minimum of the 11-year cycle to the reversal; $\mathrm{k}=2$ corresponds to the interval from the reversal to the minimum). This formula shows that from the minimum to the reversal the northern hemisphere always dominates, while from the reversal to the minimum the dominant 
E.S. Vernova et al.

Table 1. Polarity of solar magnetic fields and north-south asymmetry for Solar Cycles $21-22$

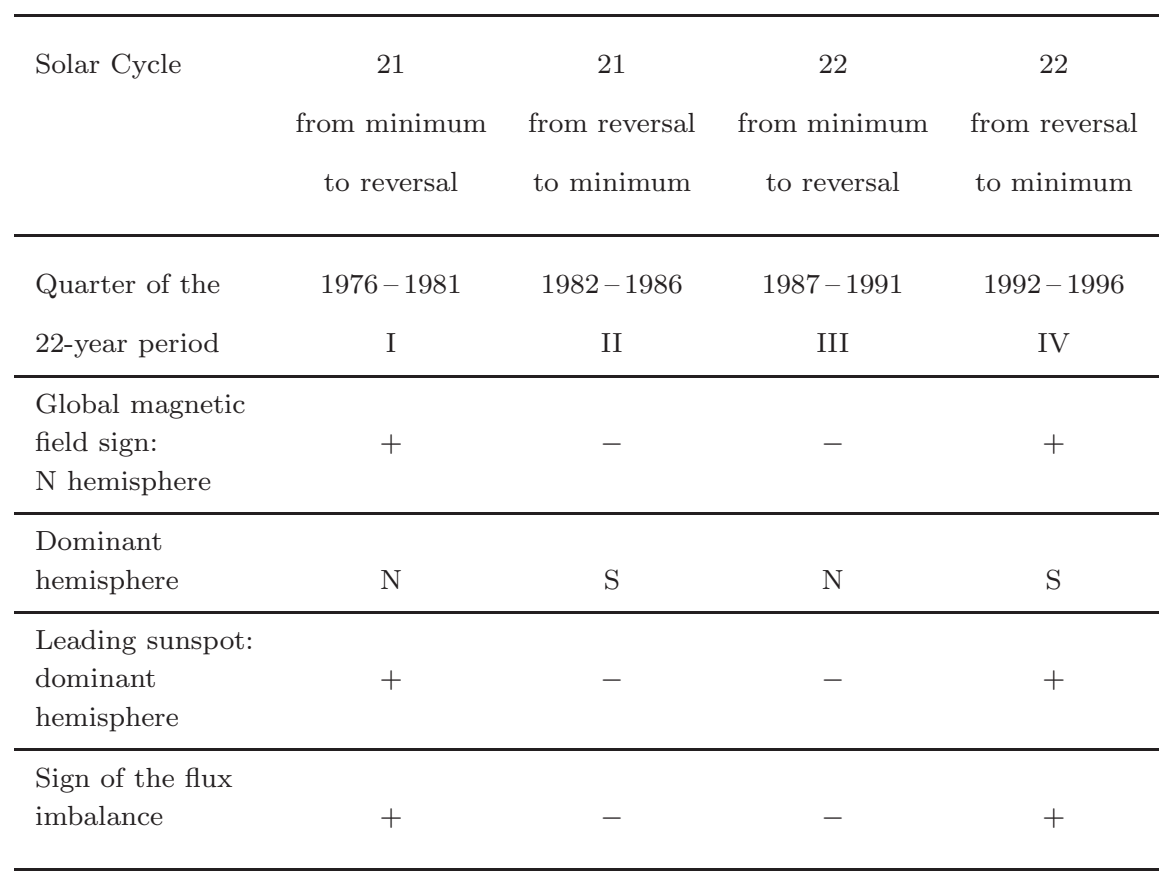

hemisphere is the southern one. We stress that this relation is obtained for Solar Cycles $21-23$ when the northern hemisphere preceded the southern one in time (see Introduction).

For the three cycles that we considered, Equation (2) correctly describes the change of the north-south asymmetry of magnetic fields shown in Figure 2 As has been stated in the Introduction, a number of works established the change of the dominant hemisphere for the ascending and descending phases of the solar cycle. This effect was observed for different manifestations of solar activity (see Vizoso and Ballester, 1987, and references in the Introduction). A rapid transition from the domination of the northern hemisphere to the domination of the southern one after the magnetic-field reversal has been observed for sunspot areas (Vernova et al., 2002) and for solar flares (Kane, 2005). A reverse change from the southern hemisphere to the northern one during the solar minimum was found for sunspots (Swinson, Koyama, and Saito, 1986). Because the change of the photospheric magnetic field determines the cyclic change of various indices of solar activity, the proposed formula can be interpreted as a generalization of some of the results cited above. 


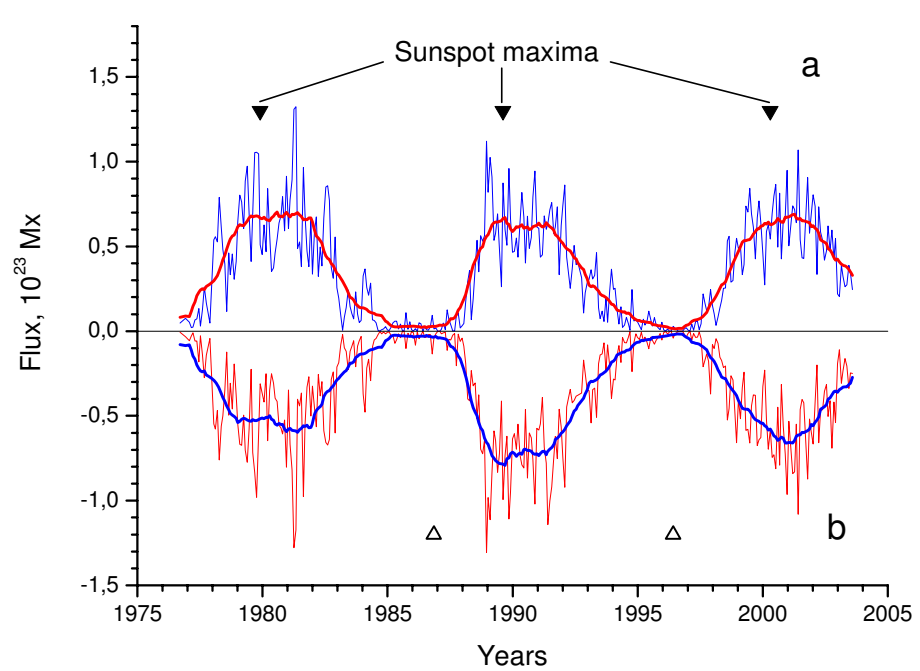

Figure 3. Fluxes of the strong magnetic fields $[B>100 \mathrm{G}]$ of the northern hemisphere (helio-latitudes from $0^{\circ}$ to $+40^{\circ}$ ): positive (a) and negative (b) photospheric magnetic fluxes. Thick lines represent data averaged over 20 solar rotations. Triangles mark solar maxima (black triangles) and solar minima (white triangles).

\section{Positive and Negative Magnetic Fields and their Imbalance for Each Hemisphere}

In the previous section, absolute values of magnetic field were used to evaluate the total magnetic flux. Now we consider the positive and negative fluxes of the photospheric magnetic field separately to study their imbalance for each of the solar hemispheres. As above, we only take into account the strong magnetic fields $(B>100 \mathrm{G})$ of the equatorial region (from $-40^{\circ}$ to $\left.+40^{\circ}\right)$. In Figure 3 positive $\left(F_{\mathrm{N}}^{\text {pos }}>0\right.$, Figure 3 $)$ and negative $\left(F_{\mathrm{N}}^{\text {neg }}<0\right.$, Figure 3b) magnetic fluxes for the northern hemisphere of the Sun are presented. Smoothed curves are shown, which were obtained using adjacent averaging over 20 rotations. It can be seen that both fluxes show a distinct 11-year periodicity. This is expected because we consider the strong fields, which are known to be connected with active regions. As a result, the magnetic flux drops almost to zero during minima of solar activity.

Positive and negative fluxes are well correlated, but their difference $\left[\Delta F_{\mathrm{N}}=\right.$ $\left.\left|F_{\mathrm{N}}^{\mathrm{pos}}\right|-\left|F_{\mathrm{N}}^{\text {neg }}\right|\right]$ (flux imbalance or net flux) shows regular changes closely connected with the phase of the 22-year solar cycle. The smoothed value of the difference $\left[\Delta F_{\mathrm{N}}\right]$ for the northern hemisphere is shown in Figure 4. Maxima of the difference amount to $\approx 20 \%$ of the average magnetic flux for the northern hemisphere. Wolf numbers are shown for comparison with the solar-cycle progression. The difference varies with the 22-year cycle and reaches extrema during maxima of solar activity. The difference is close to zero for several years around the minima of solar activity (1984- 1987 and 1995-1998). During these periods the difference passes through zero. Thus, from one minimum to another, the sign 


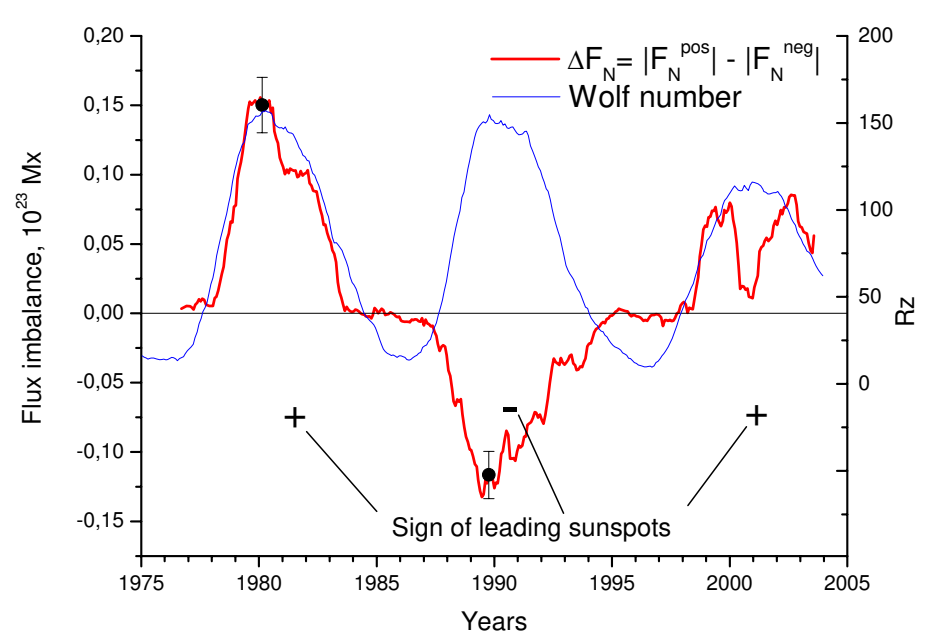

Figure 4. Positive and negative flux imbalance $\left[\Delta F_{\mathrm{N}}=\left|F_{\mathrm{N}}^{\text {pos }}\right|-\left|F_{\mathrm{N}}^{\text {neg }}\right|\right]$ (northern hemisphere) - thick red line. Wolf numbers [Rz] are plotted by the blue line. Sign of leading sunspots in the northern hemisphere for each of the three solar cycles is shown. Statistical spread of averaged data is given for extrema of the imbalance curve.

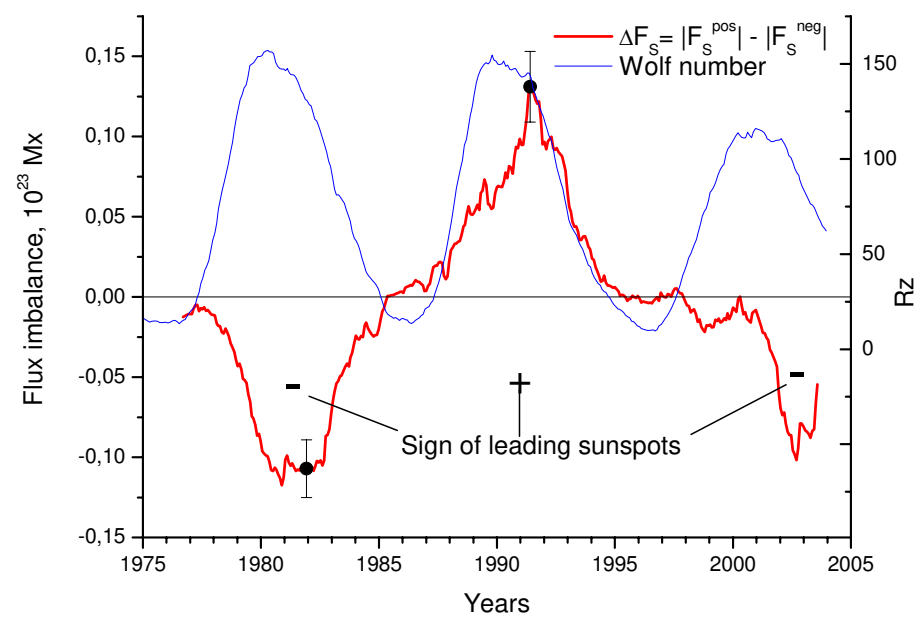

Figure 5. Positive and negative flux imbalance $\left[\Delta F_{\mathrm{S}}=\left|F_{\mathrm{S}}^{\text {pos }}\right|-\left|F_{\mathrm{S}}^{\text {neg }}\right|\right]$ (southern hemisphere) - thick red line. Wolf numbers $[\mathrm{Rz}]$ are plotted by the blue line. Sign of leading sunspots in the southern hemisphere for each of the three solar cycles is shown. Statistical spread of averaged data is given for extrema of the imbalance curve.

of $\Delta F_{\mathrm{N}}$ (the difference between positive and negative fluxes) does not change. In Figure 4 the sign of leading sunspots in bipolar sunspot groups is shown. The sign of the difference coincides with the sign of leading sunspots in the northern hemisphere. Evidently, the leading sunspots contribute more to the magnetic flux than the following ones. 
Similar results are obtained for the southern hemisphere: the sign of the imbalance always coincides with the sign of leading sunspots (Figure 5). Thus, a positive difference corresponds to the time when leading sunspots in the given hemisphere have positive polarity. In the same way, the negative difference corresponds to the period when leading sunspots have negative polarity. This effect can be observed for almost three solar cycles both for the northern and southern hemispheres of the Sun. The imbalance of positive and negative fluxes in each of the solar hemispheres shows a 22-year recurrence that is directly connected with the Hale cycle. The absolute values of the imbalance for both solar hemispheres $\left[\left|\Delta F_{\mathrm{N}}\right|,\left|\Delta F_{\mathrm{S}}\right|\right]$ closely follow the sunspot activity for Cycles 21 and 22 (Figures 4 . 5). The correlation becomes somewhat poorer for Cycle 23, although the main effect can be observed in this case as well: the change of the imbalance sign during solar minimum.

These results agree with direct measurements of the magnetic field of sunspots, which give a higher magnetic flux for the leading sunspots than for the following ones (Vitinsky, Kopecky, and Kuklin, 1986 , Choudhary, Venkatakrishnan, and Gosain, 2002; Fan, 2009). It has been shown that the balance of magnetic fluxes is not observed for all classes of sunspot groups (Vitinsky, Kopecky, and Kuklin, 1986). Moreover, the longitude-averaged magnetic field of active regions does not become zero, the sign of the field being determined by the polarity of leading sunspots (Obridko and Shel'ting, 2007). For the northern hemisphere during odd solar cycles, magnetic fields with positive polarity slightly exceed in strength the fields of the opposite sign, occupying at the same time smaller areas. Note that according to our results not only the strength $[B]$, but also the flux of positive fields in the northern hemisphere $(F=B S$, where $S$ is the sunspot area) exceeds the flux of the opposite sign during odd cycles.

The imbalance between magnetic fields of different polarities (Figures 4 and 5) may be connected with the asymmetry in morphology of the leading and following sunspots. It is well known that the leading polarity of an active region tends to be in the form of large sunspots, whereas the following polarity tends to appear more dispersed and fragmented. Numerical simulations of Fan, Fisher, and DeLuca (1993) showed that the area asymmetry, as well as the field-strength asymmetry, between the preceding and following polarities might be produced by the Coriolis force during a flux tube's rising motion in the solar convection zone. The area asymmetry of bipolar magnetic fields was studied quantitatively by Yamamoto (2012) using magnetograms of bipolar regions. Areas of the preceding polarities proved to be smaller than those of the following polarities in many bipolar magnetic regions. Only active regions with magnetic imbalances lower than $15 \%$ were selected for this study. Several authors have reported higher magnetic-flux imbalances in active regions (see Tian, Liu, and Wang, 2003, and references therein). About $47 \%$ of the active regions are in a $10 \%$ flux imbalance and $31 \%$ of the active regions are in a flux imbalance between $10 \%$ and $20 \%$. Only $22 \%$ of the active regions have a flux imbalance higher than $20 \%$ (Tian, Liu, and Wang, 2003). The lower limit of the field strength considered was set at $50 \mathrm{G}$ by Yamamoto (2012) and at $20 \mathrm{G}$ by Tian, Liu, and Wang (2003). In our study only strong magnetic fields $(B>100 \mathrm{G})$ were considered. One could argue that the magnetic-field imbalance of the leading and the following polarity 


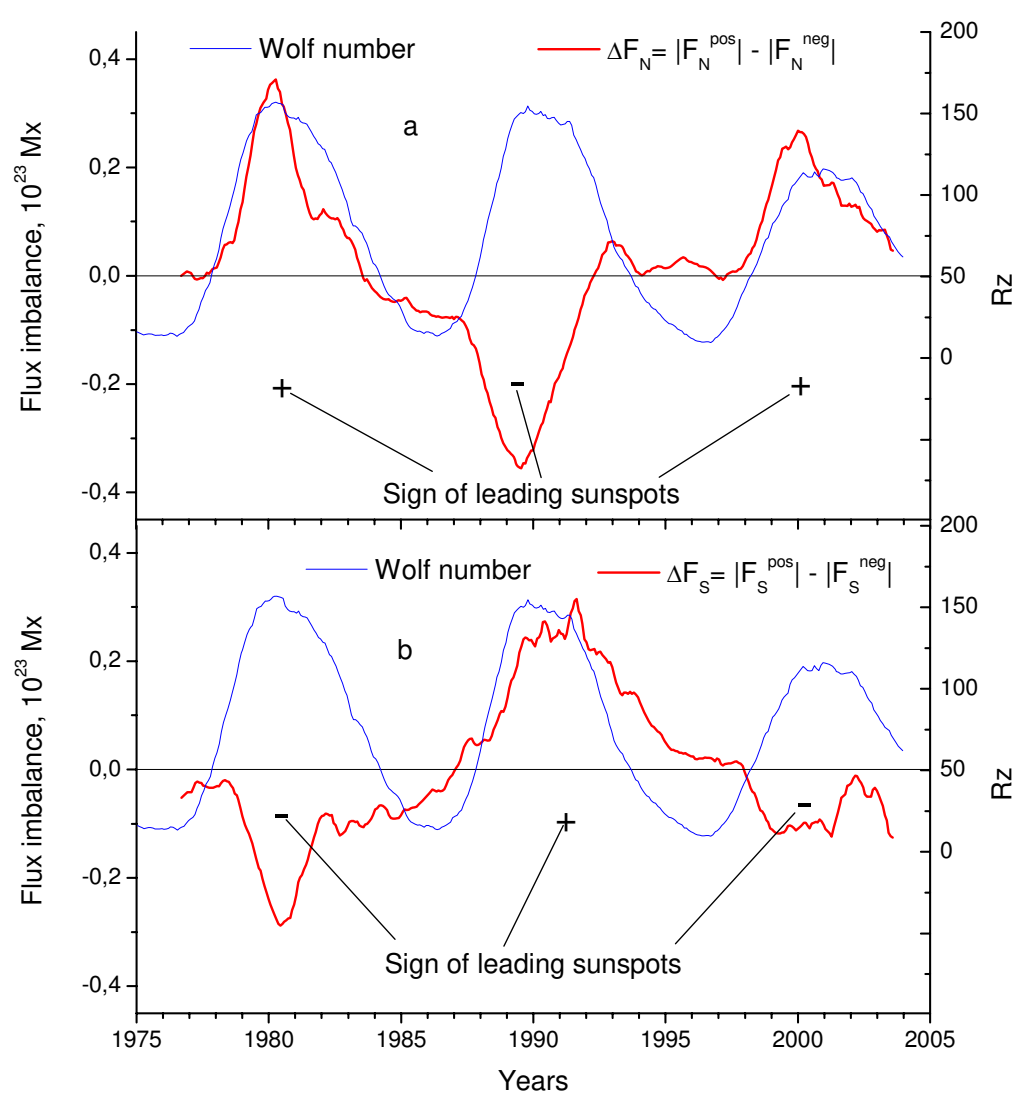

Figure 6. Positive and negative flux imbalance calculated for all values of magnetic-field strength (thick red line): (a) northern hemisphere $\left[\Delta F_{\mathrm{N}}=\left|F_{\mathrm{N}}^{\text {pos }}\right|-\left|F_{\mathrm{N}}^{\text {neg }}\right|\right]$; (b) southern hemisphere $\left[\Delta F_{\mathrm{S}}=\left|F_{\mathrm{S}}^{\text {pos }}\right|-\left|F_{\mathrm{S}}^{\text {neg }}\right|\right]$. Wolf numbers [Rz] are plotted by the blue line. Sign of leading sunspots is shown for each of the three solar cycles.

that we observed (Figures 4 and 5) arose because weaker fields of the following sunspots were not included in our calculation.

To check this possibility we extended our study of imbalance to all values of magnetic-field strength (Figure 6).

The imbalance of positive and negative fluxes proved to be even higher than for strong magnetic fields (Figures 4 and 5). This is true both for the northern and southern hemispheres. As pointed out by Svalgaard and Kamide (2013), magnetic flux from decaying sunspots moves toward the poles, predominantly from the following spots. This may lead to a deficit of the following polarity in the sunspot zone and produce the observed imbalance. 


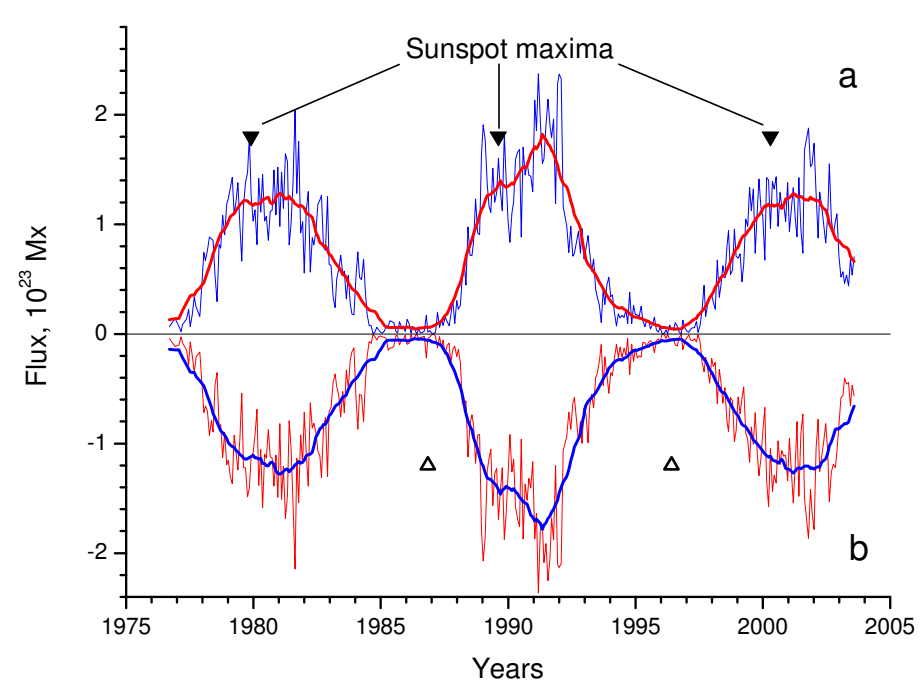

Figure 7. Positive and negative fluxes of the strong magnetic fields $[B>100 \mathrm{G}]$ for both solar hemispheres together (helio-latitudes from $-40^{\circ}$ to $+40^{\circ}$ ): positive (a) and negative (b) photospheric magnetic fluxes. Thick lines represent data averaged over 20 solar rotations. Triangles mark solar maxima (black triangles) and solar minima (white triangles).

\section{Imbalance of Positive and Negative Magnetic Fluxes for the Whole Latitude Range from $-40^{\circ}$ to $+40^{\circ}$}

Now we consider the imbalance of positive and negative magnetic fluxes for the whole latitude interval from $-40^{\circ}$ to $+40^{\circ}$. As earlier, we consider only strong magnetic fields $[B>100 \mathrm{G}]$.

Positive $\left[F_{\mathrm{N}}^{\mathrm{pos}}+F_{\mathrm{S}}^{\mathrm{pos}}\right]$ and negative $\left[F_{\mathrm{N}}^{\text {neg }}+F_{\mathrm{S}}^{\text {neg }}\right]$ fluxes are presented in Figure 7 Changes of positive and negative fluxes are very similar, both follow the 11-year solar cycle. However, the difference of these fluxes, i.e. the imbalance of positive and negative fluxes shows regular changes in the course of a 22-year solar cycle (see our results in Vernova et al., 2009). The difference between positive and negative fluxes $[\Delta F]$ is displayed in Figure 8, where

$$
\Delta F=\left|F_{\mathrm{N}}^{\mathrm{pos}}+F_{\mathrm{S}}^{\mathrm{pos}}\right|-\left|F_{\mathrm{N}}^{\mathrm{neg}}+F_{\mathrm{S}}^{\mathrm{neg}}\right| .
$$

The difference was calculated for each of the solar rotations, and then smoothed using adjacent averaging over 20 rotations. The smoothed value of the difference is shown in Figure 8 in comparison with the Wolf numbers. The difference between the fluxes depends on the level of solar activity. The difference becomes nonzero during periods of high solar activity and is almost equal to zero around minima of solar activity (1985 - 1988 and 1994-1998).

In the upper part of Figure 8, the polarity of the polar magnetic field in the northern hemisphere is displayed. Green circles mark the change of the sign of the imbalance. The imbalance changes its sign near the global magnetic field reversals. Thus, the sign of the dominant flux remains constant within 11 years 


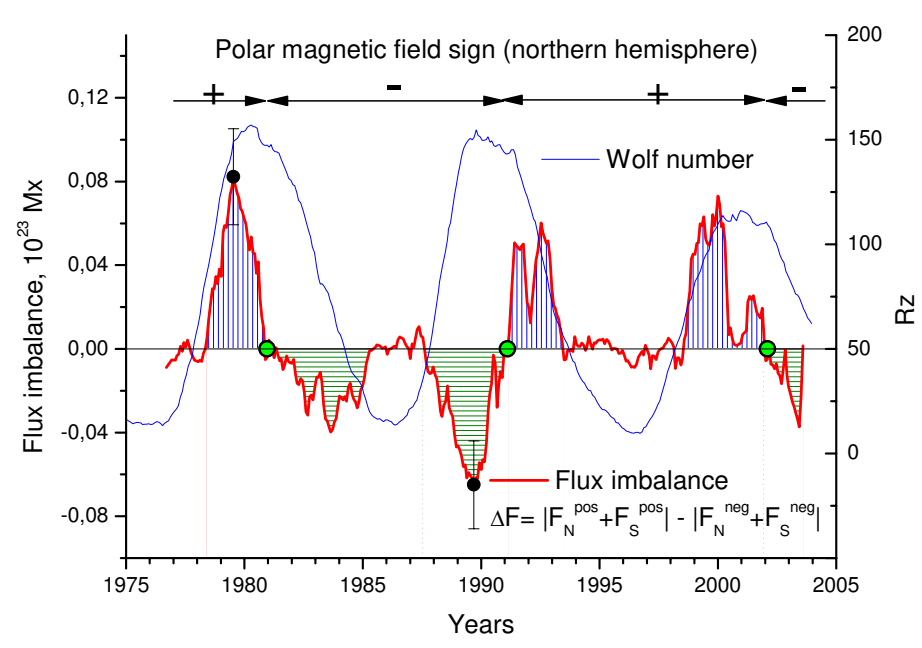

Figure 8. Positive and negative flux imbalance for both solar hemispheres $\Delta F=\left|F_{\mathrm{N}}^{\mathrm{pos}}+F_{\mathrm{S}}^{\mathrm{pos}}\right|-\left|F_{\mathrm{N}}^{\text {neg }}+F_{\mathrm{S}}^{\text {neg }}\right|-$ thick red line (helio-latitudes from $-40^{\circ}$ to $\left.+40^{\circ}\right)$. Periods of the positive polarity domination are shown by the vertical blue shading; horizontal green shading corresponds to the negative polarity domination. Green circles mark the change of the imbalance sign. Statistical spread of averaged data is given for extrema of the imbalance curve. Wolf numbers $[\mathrm{Rz}]$ are plotted by the blue line. Polar magnetic-field sign is shown for the northern hemisphere.

from one global magnetic-field reversal to the next. From the minimum of 1976 to the reversal of Cycle 21, the imbalance is positive. During the reversal of the global magnetic field (1981) it becomes negative and keeps this sign until the next reversal (1991). Then the sign of the imbalance changes and again becomes positive. The maximum of the imbalance amounts to $4 \%$ of the average magnetic flux.

As Figure 8 shows, the difference of fluxes changes with the 22-year period, passing through zero near to the global magnetic-field reversal. Thus, the difference of fluxes preserves its sign within 11 years from one reversal to another. The sign of the difference always coincides with the polar magnetic-field sign in the northern hemisphere. Before the reversal, the sign of the difference coincides not only with the polar magnetic-field sign in the northern hemisphere, but also with the sign of leading sunspots in the northern hemisphere. In contrast, after the reversal, the sign of the difference coincides with the polar magnetic-field sign in the northern hemisphere and with the sign of leading sunspots in the southern hemisphere.

The sign of the imbalance for each of the four quarters of the 22-year magnetic cycle is shown in Table 1 compared with the change of magnetic-field polarities.

The change of the sign of the positive and negative magnetic flux imbalance $\Delta F$ can be expressed by the following formula:

$$
\operatorname{sign} \Delta F=(-1)^{\mathrm{n}+\mathrm{k}},
$$

where $\mathrm{n}=1,2(\mathrm{n}=1$ corresponds to the odd solar cycle, $\mathrm{n}=2$ to the even one); $\mathrm{k}=1,2$ ( $\mathrm{k}=1$ corresponds to the interval of the 11-year cycle from the 
minimum up to the reversal; $\mathrm{k}=2$ to the interval from the reversal up to the minimum). In this way, the imbalance sign is determined by two factors: the parity of the solar cycle on one hand, and the phase of the 11-year cycle on the other hand.

\section{Imbalance of the Fluxes and the Asymmetry Between Leading and Following Sunspots}

We have seen that the sign of the imbalance of positive and negative fluxes remains constant for 11 years from one reversal to another and coincides with the sign of the polar magnetic field of the Sun in the northern hemisphere.

The question arises whether it is possible to specify one of the four magnetic fluxes $\left[F_{\mathrm{N}}^{\text {pos }}, F_{\mathrm{N}}^{\text {neg }}, F_{\mathrm{S}}^{\text {pos }}, F_{\mathrm{S}}^{\text {neg }}\right]$ that plays the dominant role and determines the sign. When we considered the imbalance of positive and negative flux in the latitude range from $-40^{\circ}$ to $+40^{\circ}$ we took into account the flux of the leading and following sunspots. Thus, the magnetic flux of positive polarity contains the contribution of the leading sunspots of one hemisphere, and the contribution of the following sunspots of the opposite hemisphere.

Because for each solar cycle the sign of leading sunspots in each of the Sun's hemispheres is known, it is possible to evaluate the imbalance of the magnetic fluxes for the leading sunspots alone. For example, for Solar Cycle 21 leading sunspots in the northern hemisphere have a positive polarity $\left[F_{\mathrm{N}}^{\mathrm{pos}}>0\right]$, while leading sunspots of the southern hemisphere have a negative sign $\left[F_{\mathrm{S}}^{\text {neg }}<0\right]$. This means that the difference $\left[\Delta F_{\mathrm{L}}=\left|F_{\mathrm{N}}^{\text {pos }}\right|-\left|F_{\mathrm{S}}^{\text {neg }}\right|\right]$ represents the imbalance of the magnetic flux of leading sunspots during Cycle 21. An analogous approach can be used for other solar cycles. The resulting imbalance of the leading sunspot fluxes is shown in Figure 9,

All of the basic features of the total imbalance (Figure 8) are also observed in the imbalance of the leading sunspot fluxes (Figure 9). The sign of the dominant flux changes during the global magnetic-field reversal and then remains constant for 11 years until the next reversal. Similar to the net flux (Figure 8), the imbalance of the leading sunspot fluxes (Figure 9) is positive from the solar minimum to the reversal of Cycle 21; around the time of the reversal of the global magnetic field it becomes negative and retains this sign until the next reversal (Figure 9). Then the sign of the imbalance changes and again becomes positive.

It should be noted that the imbalance of leading sunspot flux is significantly higher than the total imbalance that includes all four fluxes $\left[F_{\mathrm{N}}^{\text {pos }}, F_{\mathrm{N}}^{\text {neg }}, F_{\mathrm{S}}^{\text {pos }}\right.$, $\left.F_{\mathrm{S}}^{\text {neg }}\right]$. The maxima of the leading-sunspot imbalance amount to $\approx 15 \%$ of the average magnetic flux. This effect occurs because the imbalance of the following sunspots is in antiphase with the imbalance of leading sunspots (Figure 10). Moreover, the imbalance of leading and of following sunspots is related by an almost linear dependence with the correlation coefficient $R=-0.98$. This means that when the leading sunspots of one hemisphere dominate over the leading sunspots of the other hemisphere, the following sunspots in the first hemisphere dominate over the following sunspots of the other hemisphere.

The imbalance between flux of leading sunspots in the two hemispheres maintains its sign for each quarter of a 22-year magnetic cycle. The same is true 


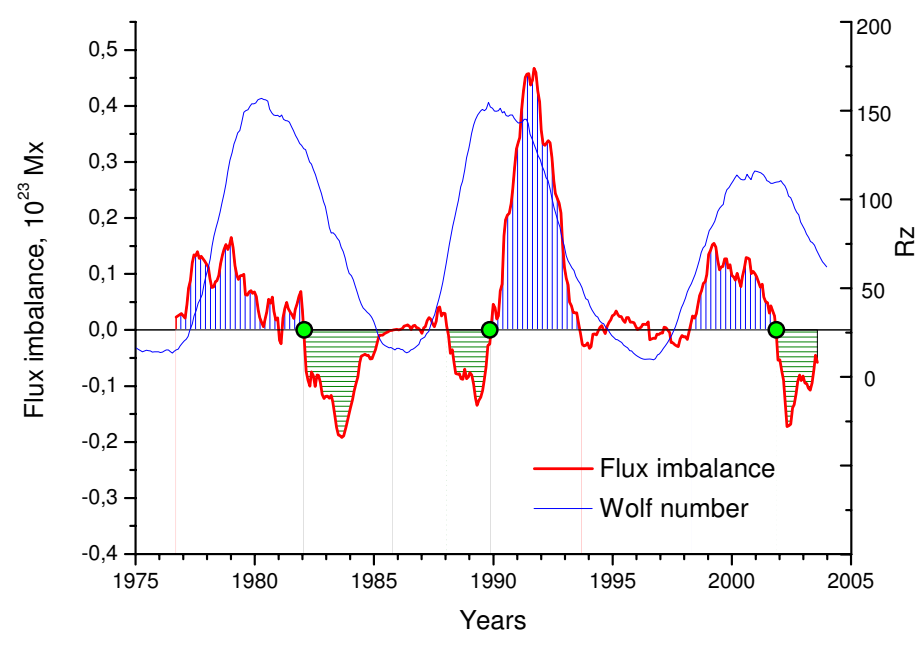

Figure 9. Positive and negative flux imbalance of leading sunspots for both solar hemispheres $\left[\Delta F_{\mathrm{L}}=\left|F_{\mathrm{N}}^{\text {pos }}\right|-\left|F_{\mathrm{S}}^{\text {neg }}\right|\right]$ - thick red line (helio-latitudes from $-40^{\circ}$ to $\left.+40^{\circ}\right)$. Periods of the positive polarity domination are shown by the vertical blue shading; horizontal green shading corresponds to the negative polarity domination. Green circles mark the change of the imbalance sign. Wolf numbers $[\mathrm{Rz}]$ are plotted by the blue line.

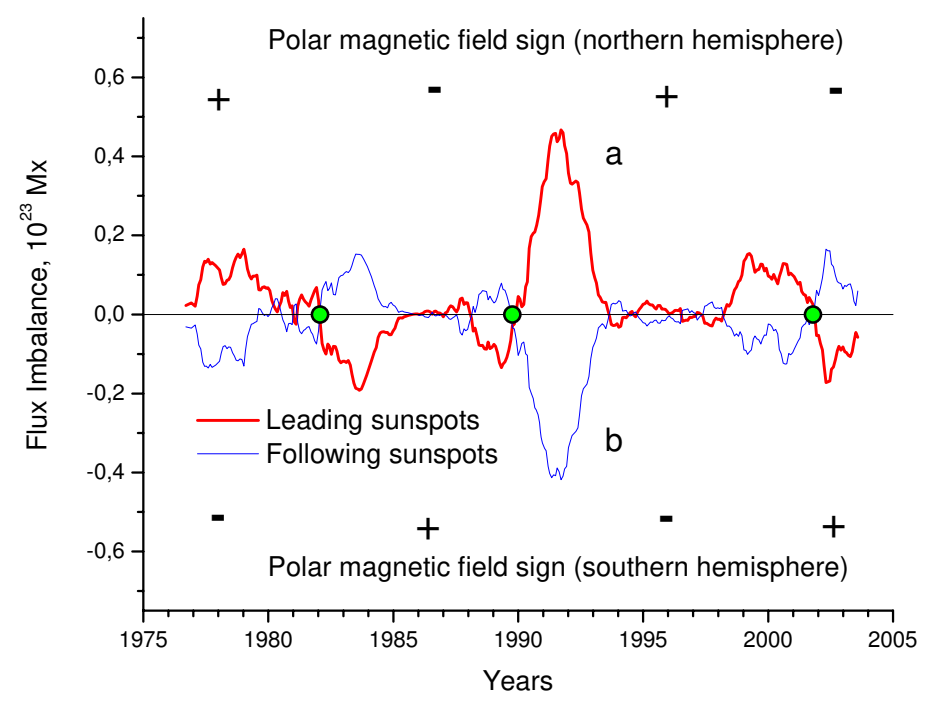

Figure 10. Flux imbalance of leading (thick red line) and following (blue line) sunspots of both hemispheres. Polar magnetic-field sign for the northern (respectively, southern) hemisphere is shown in the upper (respectively, lower) part of the figure. 

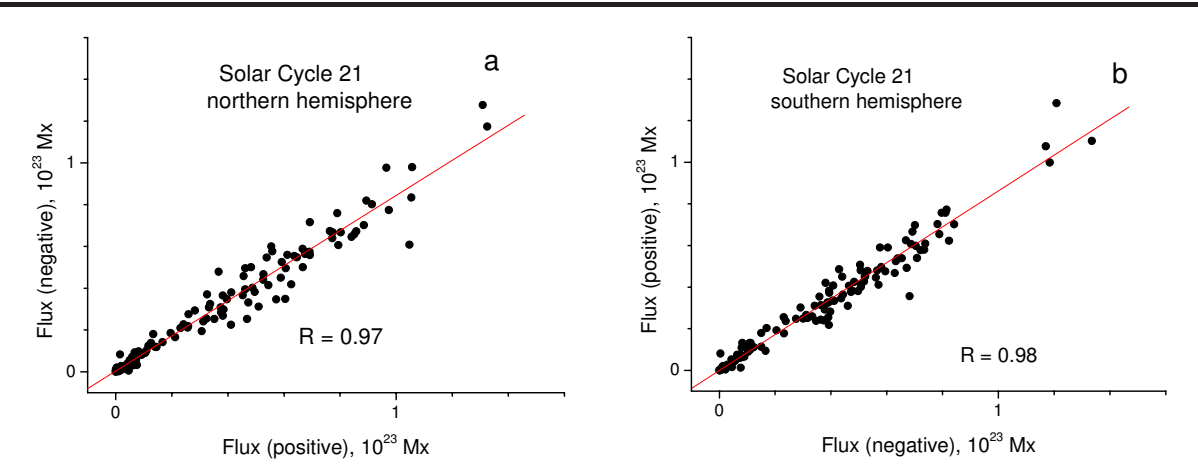

Figure 11. Correlation of positive and negative magnetic fluxes (Solar Cycle 21): (a) northern hemisphere: $F_{\mathrm{N}}^{\mathrm{pos}}$ and $F_{\mathrm{N}}^{\text {neg }} ;(\mathrm{b})$ southern hemisphere: $F_{\mathrm{S}}^{\text {neg }}$ and $F_{\mathrm{S}}^{\text {pos }}$. Regression line is shown in red. R: correlation coefficient. Each point represents one Carrington rotation.

for the following sunspots, so that the northern hemisphere dominates from a minimum to a reversal, and the southern hemisphere dominates from a reversal to a minimum for both leading and following sunspots. Simultaneous domination of the leading and following sunspot fluxes in one of the hemispheres results in the north-south asymmetry of the solar magnetic flux. During the period of low activity of the Sun, the imbalance is close to zero. It changes sign around the reversal of the polar magnetic field (Figure 10).

The sign of the imbalance of leading sunspots (Figure 10) does not change during 11 years from one reversal to the next and coincides with the sign of the polar magnetic field in the northern hemisphere (shown at the top of Figure 10). The sign of the imbalance of following sunspots does not change either during the 11 years from one reversal to the next and coincides with the sign of the polar magnetic field in the southern hemisphere (shown at the bottom of Figure [10).

The absolute values of the leading and following sunspot fluxes in the same hemisphere are very well correlated. For Solar Cycle 21, the flux of the following sunspots is plotted against the leading sunspot fluxes in Figure 11] (northern hemisphere) and in Figure 11] (southern hemisphere). The correlation coefficients are 0.97 and 0.98 , respectively. The slope of the regression line is $0.84 \pm 0.02$ for the northern hemisphere (Figure 117) and $0.86 \pm 0.02$ for the southern one (Figure 11]). The slopes for the two hemispheres coincide within the uncertainties. The deviation of this slope from unity is a measure of the leading-following asymmetry of the flux concentration. Thus for the strong magnetic fields $[B>100 \mathrm{G}]$ the field-strength asymmetry between the preceding and following polarities is nearly the same for the northern and southern hemispheres. The correlation becomes much poorer if one considers sunspots belonging to the opposite solar hemispheres. For the same cycle, the following-sunspot fluxes (southern hemisphere) are plotted against those of the leading-sunspot fluxes (northern hemisphere) in Figure 12. The scatter of points is much greater, and the correlation coefficient is only 0.53 .

These observations are summarized in Table 2 where correlation coefficients are given for different combinations of the flux pairs (positive and negative fluxes, 
northern and southern hemispheres). Correlation coefficients between the leading and following sunspots that belong to the same hemisphere show very high values. All correlation coefficients between the fluxes of different hemispheres are much lower, varying from 0.52 to 0.58 .

Table 2. Correlation coefficients for magnetic fluxes of Solar Cycle 21

\begin{tabular}{lccc}
\hline Magnetic fluxes & $F_{\mathrm{N}}^{\text {neg }}$ & $F_{\mathrm{S}}^{\text {neg }}$ & $F_{\mathrm{S}}^{\text {pos }}$ \\
\hline$F_{\mathrm{N}}^{\text {pos }}$ & 0.97 & 0.58 & 0.56 \\
$F_{\mathrm{N}}^{\text {neg }}$ & & 0.53 & 0.52 \\
$F_{\mathrm{S}}^{\text {neg }}$ & & & 0.98 \\
\hline
\end{tabular}

Domination of leading sunspots of one of the hemispheres over leading sunspots of the other hemisphere is, therefore, accompanied by domination of the following sunspots of the same hemisphere. But the polarity of the leading and following sunspots situated in the same hemisphere is opposite, therefore when we take into account both types of sunspots (Figure 8), we observe an imbalance lower than that of the leading sunspots alone (Figure 91).

Thus, the observed effect of the magnetic-flux imbalance and its change should be attributed to the major contribution of the leading sunspot flux, which exceeds the contribution of the following sunspots and determines the resulting sign of the effect.

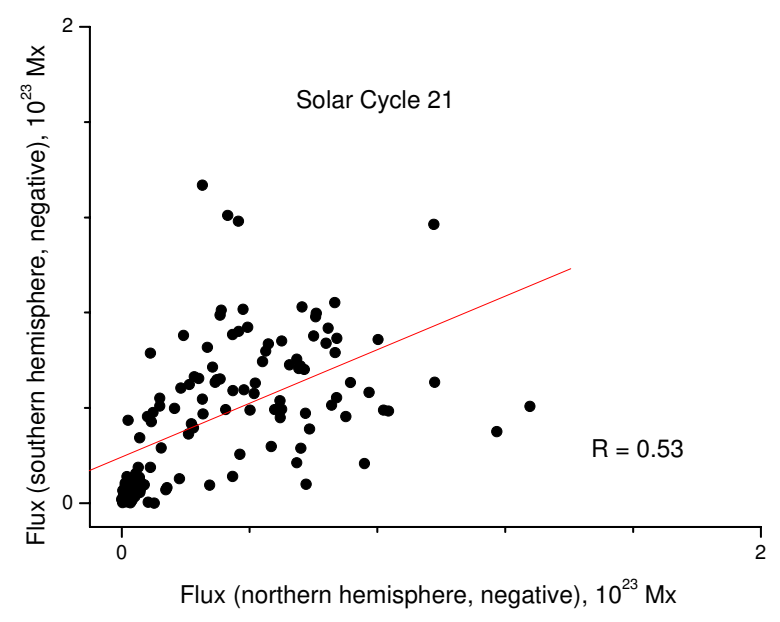

Figure 12. Correlation of negative magnetic fluxes of the northern $\left[F_{\mathrm{N}}^{\text {neg }}\right]$ and southern $\left[F_{\mathrm{S}}^{\text {neg }}\right]$ hemispheres (Solar Cycle 21). Regression line is shown in red. R: correlation coefficient. Each point represents one Carrington rotation. 
The following conclusions can be drawn on the basis of Figures 8 9, and 10:

i) The sign of the positive and negative flux imbalance remains constant for 11 years from one reversal of the global magnetic field to the next, coinciding with the sign of the polar field in the northern hemisphere (Figure 8).

ii) The sign of the imbalance is determined by the prevalence of the leadingsunspot flux in one of the hemispheres over the leading-sunspot flux of the opposite hemisphere (Figure 9).

iii) If leading sunspots of a certain hemisphere dominate leading sunspots of the opposite hemisphere, then the following sunspots of the first hemisphere will dominate the following sunspots of the second one (Figure 10).

North-south asymmetry and imbalance of positive and negative magnetic fluxes represent different, yet closely connected, features of the photospheric magnetic field. The north-south asymmetry (as defined by Equation (1D) is the difference of the total fluxes of the two solar hemispheres. In contrast, the imbalance of positive and negative magnetic fluxes represents the net flux of both hemispheres (Equation (3)).

The regular change of the magnetic flux north-south asymmetry for Solar Cycles $21-23$ described in Section 3 can be deduced from conclusions i)-iii). We consider successively the four quarters of a 22-year magnetic cycle of the Sun (Table 1).

For the first quarter $(1976-1981)$, the imbalance was positive $[\Delta F>0]$ (see Table 1 and Figure (8) because of the major contribution of leading sunspots of the northern hemisphere, which have a positive sign during this period $\left[F_{\mathrm{N}}^{\text {pos }}>0\right]$ (Figure 9). Both positive and negative fluxes (leading and following sunspots) of the northern hemisphere (Figure 10) exceed the corresponding fluxes of the leading and following sunspots of the opposite hemisphere $\left[\left|F_{\mathrm{N}}^{\text {pos }}\right|>\left|F_{\mathrm{S}}^{\text {neg }}\right|\right.$ and $\left.\left|F_{\mathrm{N}}^{\text {neg }}\right|>\left|F_{\mathrm{S}}^{\text {pos }}\right|\right]$, which means that the total magnetic flux of the northern hemisphere will dominate.

For the second quarter (1982-1986), the sign of the imbalance changes $[\Delta F<0]$; now it is determined by leading sunspots of negative sign, i.e. by leading sunspots of the southern hemisphere. Both the leading and following sunspots of the southern hemisphere dominate the corresponding sunspots of the northern hemisphere. As a result, after the reversal the total flux of the southern hemisphere will dominate.

For the third quarter (1987-1991), the imbalance preserves the negative sign, but after solar minimum, leading sunspots of the negative sign appear in the northern hemisphere. Thus, a negative sign of the imbalance points to the domination of leading sunspots of the northern hemisphere. The magnetic fluxes of leading (following) sunspots in the northern hemisphere exceed the corresponding fluxes of the southern hemisphere. Now the total flux of the northern hemisphere will dominate.

For the fourth quarter (1992-1996), the sign of the imbalance changes and becomes positive. It corresponds to the sign of leading sunspots of the southern hemisphere. Domination of leading (and following) sunspots of the southern hemisphere results in the prevalence of the total magnetic flux of the southern hemisphere.

In this way, the magnetic flux imbalance and the north-south asymmetry for Solar Cycles $21-23$ proved to be linked. 


\section{Conclusions}

We were concerned here with the photospheric magnetic fields connected with solar active regions. Therefore, we confined ourselves to the study of the nearequatorial helio-latitudes $\left( \pm 40^{\circ}\right)$ and strong magnetic fields $(B>100 \mathrm{G})$. For the northern and southern hemispheres, fluxes of positive and of negative photospheric magnetic fields were evaluated to obtain four resulting fluxes: $F_{\mathrm{N}}^{\text {pos }}$, $F_{\mathrm{N}}^{\text {neg }}, F_{\mathrm{S}}^{\text {pos }}$, and $F_{\mathrm{S}}^{\text {neg }}$. Since we considered the sunspot region and only took into account strong fields, the main contribution to the flux of positive (negative) polarity for each hemisphere could be attributed to the flux of the leading or the following sunspots with the corresponding polarities. Thus, each of the four fluxes may be considered as the flux of leading (following) sunspots in one of the hemispheres. The imbalance of the fluxes of positive and negative polarities manifests itself in two forms: as the imbalance of the fluxes within one hemisphere, and as the total imbalance for both hemispheres.

i) Imbalance of the fluxes for an individual hemisphere. We showed that for the leading and following sunspots of one hemisphere, the moduli of their fluxes (for Solar Cycle 21) are very strongly correlated (the correlation coefficient is 0.97 for the northern hemisphere and 0.98 for the southern hemisphere), in contrast to the fluxes of the sunspots from different hemispheres (the correlation coefficient changes from 0.52 to 0.58 ). However, the imbalance of the positive and negative fluxes of the solar hemisphere shows significant (20\%) systematic changes during the 22-year cycle. The sign of the difference between the positive and negative fluxes is constant from one minimum to the next and coincides with the sign of leading sunspots in the corresponding hemisphere, which implies the domination of the leading-sunspot flux over the flux of the following sunspots (Figures 4 and 5). Thus, the sign of imbalance is directly related to the Hale-sign-law cycle.

ii) Total imbalance. For the whole interval of helio-latitudes from $+40^{\circ}$ to $-40^{\circ}$ the difference between the positive and the negative fluxes ( $4 \%$ of the average magnetic flux) also displays a 22-year periodicity; however, in contrast to any separately considered hemisphere, the sign of the total imbalance changes during the period of the Sun's global magnetic-field reversal (Figure 8). The sign of the imbalance does not change during the 11 years from one reversal to the next and always coincides with the sign of the Sun's polar magnetic field in the northern hemisphere. We showed that the imbalance of the magnetic fluxes of the leading sunspots $(15 \%)$ has the same sign as the total imbalance (Figure 9) and is in antiphase with the imbalance of the fluxes of the following sunspots (Figure 10). Thus, the domination of the leading sunspots in one of the hemispheres determines the sign of the magnetic flux imbalance.

The sign of the imbalance between fluxes of leading sunspots of two hemispheres changes with a 22-year magnetic cycle in the same way as the sign of the polar magnetic field in the northern hemisphere, while the imbalance between fluxes of following sunspots follows in sign the polar magnetic field in the southern hemisphere (Figure 10).

The sign of the imbalance between fluxes of leading sunspots of the two hemispheres remains constant for each quarter of a 22-year magnetic cycle (Figure10). The imbalance of the following sunspot fluxes is in antiphase with the imbalance 
of the leading sunspot fluxes. This means that domination of leading sunspots of a certain polarity is accompanied by the domination of following sunspots with opposite polarity in the same hemisphere.

These features of the magnetic-flux imbalance can explain the north-south asymmetry of the magnetic flux and its change in the course of an 11-year solar cycle. The north-south asymmetry of the magnetic flux displays a regular alternation of the dominant hemisphere for Solar Cycles 21-23 (see Figure2 and Table 11. In the ascending phase (from solar-activity minimum to the reversal) the northern hemisphere always dominates. During the reversal the dominant role passes to the southern hemisphere, which prevails until the next minimum. Near the solar-activity minimum, the northern hemisphere becomes dominant again. Data considered in our analysis $(1976$ - 2003) refer to the four Schwabe Cycles $(20-23)$, when activity of the northern hemisphere was leading in time (see Introduction). The regular change of the north-south asymmetry of the magnetic flux that we observed agrees with this effect.

The domination of the magnetic flux with a certain polarity displays a 22-year periodicity; this polarity always coincides with the polar magnetic-field polarity of the northern hemisphere.

The domination of leading sunspots in one of the hemispheres determines the sign of the magnetic-flux imbalance. At the moment of the reversal, the relative roles of leading sunspots change with the subsequent domination of leading sunspots with opposite polarity. During the solar-activity minimum, when the leading and following sunspots change their signs according to Hale's law, domination of leading sunspots of a certain polarity remains. Before the minimum, leading sunspots of the southern hemisphere dominate, while after the minimum the leading sunspots of the northern hemisphere play the dominant role. Thus during the 22-year magnetic solar cycle we observe four periods with stable configurations of magnetic fields, i.e. periods (quarters) of constant polarities of local and global magnetic fields: from the minima, where the sunspot sign changes following Hale's law, to the reversals of the Sun's global magnetic field, and from the reversals to the minima.

We showed that both the sign of the north-south asymmetry of the magnetic fluxes for Solar Cycles 21-23 and the sign of the imbalance of the positive and the negative fluxes are related to one of the quarters of the 22-year magnetic cycle where the magnetic configuration of the Sun remains constant. Our results enable us to express the change of the sign of the north-south asymmetry and for the sign of the magnetic-flux imbalance simply by Equations (2) and (4). The sign of the north-south asymmetry only depends on the phase of the 11year cycle (before or after the reversal). On the other hand, the imbalance sign depends both on the phase of the 11-year cycle and on the parity of the solar cycle. It follows that the asymmetry of the magnetic-field distribution develops according to a regular pattern and is closely related to the evolution of the local and global magnetic fields.

Acknowledgements NSO/Kitt Peak data used here are produced cooperatively by NSF/NSO, NASA/GSFC, and NOAA/SEL. We thank E.V. Miletsky for stimulating discussions and the referee for many helpful comments. 


\section{References}

Ballester, J.L., Oliver, R., Carbonell, M.: 2005, Astron. Astrophys., 431, L5-L8. ADS. DOI Bray, R.J., Loughhead, R.E.: 1964, In: Lovell, B., Kopal, Z. (eds.) Sunspots, Chapman and Hall Ltd., 232. ADS

Carbonell, M., Terradas, J., Oliver, R., Ballester, J.L.: 2007, Astron. Astrophys 476, 951. ADS DOI

Charbonneau, P.: 2010, Living Rev. Solar Phys. 7, 3. ADS DOI

Choudhary, D.P., Venkatakrishnan, P., Gosain, S.: 2002, Astrophys. J. 573, 851. ADS. DOI.

Curto, J.J., Gaya-Pique, L.R.: 2009, J. Atmos. Solar-Terr. Phys. 71, 1695. ADS DOI

Deng, L.H., Gai, N., Tang, Y.K., Xu, C.L., Huang, W.J.: 2013, Astrophys. Space Sci. 343, 27. ADS DOI

Fan, Y., Fisher, G.H., DeLuca, E.E.: 1993, Astrophys. J. 405, 390. ADS DOI

Fan, Y.: 2009, Living Rev. Solar Phys. 6, No. 4. ADS DOI

Gnevyshev, M.N., Ohl, A.L.: 1948, Astron. Zh. 25, 18 (In Russian).

Kane, R.P.: 2005, J. Atmos. Solar-Terr. Phys. 67, 429. ADS DOI

Kotov, V.A.: 2009, Bull. Crimean Astrophys. Obs. 105, 45. ADS DOI

Li, K.J., Gu, X.M.: 2000, Astron. Astrophys. 353, 396. ADS

Minarovjech, M., Rušin, V., Saniga., M.: 2008, Solar Phys. 248, 167. ADS DOI

Muraközy, J., Ludmány, A.: 2012, Mon. Not. Roy. Astron. Soc. 419, 3624. ADS DOI.

Mursula, K., Hiltula, T.: 2003, Geophys. Res. Lett. 30(22), SSC 2-1. ADS. DOI

Newton, H.W., Milsom, A.S.: 1955, Mon. Not. Roy. Astron. Soc. 115, 398. ADS

Obridko, V.N., Shel'ting, B.D.: 2007, Astron. Reports 51, 339. ADS DOI

Pulkkinen, P.J., Brooke, J., Pelt, J., Tuominen, I.: 1999, Astron. Astrophys. 341, L43. ADS.

Roy, J.R.: 1977, Solar Phys. 52, 53. ADS DOI.

Skirgiello, M.: 2005, Ann. Geophys. 23, 3139. ADS DOI

Solanki, S.K.: 2003, Astron. Astrophys. Rev. 11, 153. ADS. DOI

Svalgaard, L., Kamide, Y.: 2013, Astrophys. J. 763, 23. ADS DOI.

Swinson, D.B., Koyama, H., Saito, T.: 1986, Solar Phys. 106, 35. ADS DOI

Swinson, D.B., Shea, M.A., Humble, J.E.: 1986, J. Geophys. Res. 91, 2943. ADS DOI

Swinson, D.B., Humble, J.E., Shea, M.A., Smart, D.F.: 1991, J. Geophys. Res. 96, 1757. ADS DOI

Sykora, J., Rybak, J.: 2010, Solar Phys. 261, 321. ADS. DOI

Tian, L., Liu, Y., Wang, H.: 2003, Solar Phys. 215, 281. ADS DOI

van Driel-Gesztelyi, L., Petrovay, K.: 1990, Solar Phys. 126, 285. ADS DOI

Verma, V.K.: 2009, in Dikpati, M.; Arentoft, T.; González Hernández, I.; Lindsey, C.; Hill, F. (eds.), Solar-Stellar Dynamos as Revealed by Helio- and Asteroseismology: GONG 2008/SOHO 21 CS-416 Astron. Soc. Pac. 483. ADS

Vernova, E.S., Tyasto, M.I., Baranov, D.G.: 2007, Solar Phys. 245, 177. ADS DOI

Vernova, E.S., Mursula, K., Tyasto, M.I., Baranov, D.G.: 2002, Solar Phys. 205, 371. ADS DOI

Vernova, E.S., Mursula, K., Tyasto, M.I., Baranov, D.G.: 2004, Solar Phys. 221, 151. ADS DOI

Vernova, E.S., Tyasto, M.I., Danilova, O.A., Baranov, D.G.: 2009, in Dachev, T., Danov, D. (eds.), Fundamental Space Research, Bulgarian Acad. Sci. 88

Vitinsky, Yu.I., Kopecky, M., Kuklin, G.V.: 1986, in Dagaev, M.M. (ed.), Statistics of SunspotFormation Activity, Nauka, Moscow (In Russian), 32.

Vizoso, G., Ballester, J.L.: 1987, Solar Phys. 112, 317. ADS. DOI

Vizoso, G., Ballester, J.L.: 1990, Astron. Astrophys. 229, 540. ADS ADS

Waldmeier, M.: 1957, Z. Astrophys. 43, 149. ADS

Waldmeier, M.: 1971, Solar Phys. 20, 332. ADS DOI

Wang, Y.M., Robbrecht, E.: 2011, Astrophys. J. 736, 136. ADS DOI

Yadav, R.S., Badruddin, Kumar, S.: 1980, Indian J. Radio Space Phys. 9, 155. ADS

Yamamoto, T.T.: 2012, Astron. Astrophys. 539, A13. ADS DOI

Zolotova, N.V., Ponyavin, D.I.: 2006, Astron. Astrophys., 449, L1. ADS DOI

Zolotova, N.V., Ponyavin, D.I.: 2009, in Strassmeier, K.G. Kosovichev, A.G., Beckman, J.E. (eds.) Cosmic Magnetic Fields: From Planets, to Stars and Galaxies, IAU Symp. 259, Cambridge Univ. Press 237. ADS DOI.

Zolotova, N.V., Ponyavin, D.I., Arlt, R., Tuominen I.: 2010, Astron. Nachr. 331, 765. ADS DOI 